\title{
Evaluation of Unconventional Protein Secretion by Saccharomyces cerevisiae and other Fungi
}

\author{
Natsuko Miura ${ }^{1, *(10)}$ and Mitsuyoshi Ueda ${ }^{2}$ \\ 1 Graduate School of Life and Environmental Sciences, Osaka Prefecture University, Sakai 599-8531, Japan \\ 2 Graduate School of Agriculture, Kyoto University, Kyoto 606-8502, Japan; miueda@kais.kyoto-u.ac.jp \\ * Correspondence: miura.natsuko@biochem.osakafu-u.ac.jp; Tel.: +81-72-254-9730
}

Received: 31 July 2018; Accepted: 27 August 2018; Published: 31 August 2018

\begin{abstract}
Development of proteome analysis of extracellular proteins has revealed that a wide variety of proteins, including fungal allergens are present outside the cell. These secreted allergens often do not contain known secretion signal sequences. Recent research progress shows that some fungal allergens are secreted by unconventional secretion pathways, including autophagy- and extracellular-vesicle-dependent pathways. However, secretion pathways remain unknown for the majority of extracellular proteins. This review summarizes recent data on unconventional protein secretion in Saccharomyces cerevisiae and other fungi. Particularly, methods for evaluating unconventional protein secretion are proposed for fungal species, including S. cerevisiae, a popular model organism for investigating protein secretion pathways.
\end{abstract}

Keywords: protein secretion; Saccharomyces cerevisiae; unconventional secretion pathway; fungal allergens

\section{Introduction}

Protein secretion is an essential way for cells to communicate with extracellular environments and/or other cells. In fungi, proteins transported to extracellular space sometimes work as allergens [1-3] or virulent factors [4,5]. Previously reported fungal allergens include a number of secreted proteins [1]. Some fungal protein allergens do not contain known secretory signal peptides (SPs) as used in the conventional protein secretion (CPS) pathway [6,7]. These proteins without SPs have been regarded as intracellular allergens [1]. Recent advances in secretome analysis have revealed that metabolic enzymes and heat shock proteins (HSPs) are secreted without known SPs, suggesting important roles of non-CPS pathways in fungal pathogenesis [8-14]. In this review, first, we provide an overview on conventional and unconventional protein secretion (UPS) machineries, as well as on proteins secreted via UPS studied in several fungi, including Saccharomyces cerevisiae. Detailed molecular machineries of CPS and UPS can be found elsewhere in several excellent reviews [15-17]. Next, we discuss approaches to evaluate UPS in fungi. In addition, we present an overview on currently available proteome databases of unconventionally secreted proteins, and use of the databases. Finally, we summarize unconventionally secreted fungal allergens based on previous secretome data.

\section{Overview on Conventional and Unconventional Protein Secretions in Fungal Cells}

While the CPS machinery is well documented in fungi, the UPS machinery is less well known since there might be several UPS pathways, possibly independent of each other. For some proteins, including Acyl-CoA-binding protein Acb1p, superoxide dismutase Sod1p, and the glycolytic enzyme enolase, novel unconventional secretion pathways are studied. There are also other unconventionally secreted proteins, including which are validated to be secreted. Further research is awaited to reveal the machineries of UPS. 


\subsection{Conventional Protein Secretion in Fungal Cells}

The CPS pathway is a vesicle-mediated protein transport pathway through which proteins are translocated from the endoplasmic reticulum (ER) and Golgi towards secretion vesicles before their extra-cellular secretion. After or in the course of translation, proteins are translocated to protein transporter complexes on the ER membrane [15].

The presence of an N-terminal SP is the most significant difference between conventionally and unconventionally secreted proteins. The $\mathrm{N}$-terminal sequence is used for transporting the protein to the ER membrane and is cleaved after translocation into the ER [15]. Using SignalP [18,19], PSort [20,21], TargetP [22,23], or relevant software, the secretion signal sequence is predictable and it is easier to predict conventionally secreted proteins based on the fungal genome or using transcriptome data [24].

\subsection{Machineries of Unconventional Protein Secretion in Fungal Cells}

Proteins secreted via the UPS pathway are extracellular proteins that do not contain known SPs. Vesicle-mediated secretion is the most investigated pathway for UPS in fungal cells [25]. Given that most of the major pathogenic fungal genomes are sequenced [26] and methods for isolating intercellular vesicles are available $[27,28]$, molecular machineries for UPS have been proposed in several fungal species. For example, cytoplasmic proteins, including HSP70 and enolase were detected in extracellular vesicles isolated from Candida albicans [11], Cryptococcus neoformans [29], and Paracoccidioides brasiliensis [30], as well as in S. cerevisiae [31]. As the roles of extracellular vesicles in fungal infection were recognized [32-34], some studies employed comparative analysis of the secretome of pathogenic fungi and S. cerevisiae $[9,30]$. Vallejo and colleagues identified 72 P. brasiliensis proteins from extracellular vesicles commonly found in at least two other fungal species [30]. Among these 72 P. brasiliensis proteins, 67 were identified previously in S. cerevisiae extracellular vesicles [31], suggesting common secretion machineries among fungal species.

Bilayered extracellular vesicles in fungal species were characterized previously [9]. In S. cerevisiae, the diameters of extracellular vesicles range from 50 to $250 \mathrm{~nm}$ [31]. In 2015, Kabani and Melki reported vesicles with diameters ranging from 30 to $100 \mathrm{~nm}$ containing the fungal prion protein Sup35p [35]. Meanwhile, several types of extracellular vesicles have been described $[25,36]$. They include exosomes secreted via fusion of the multivesicular body with the plasma membrane [31], microvesicles budding from cytoplasm [37], and Golgi-involved production of membrane vesicles [31], in which vesicles may be transferred through other membrane structures such as early or late endosomes, as seen in Figure 1.

Whether a protein is secreted via a single pathway and how the secreted vesicles pass through the fungal cell wall remains unknown [25,38]. It should be noted that some extracellular vesicle-dependently secreted proteins, including HSP70 and enolase, were also found in non-vesicular fractions in P. brasiliensis [30], suggesting that single proteins are secreted via multiple unconventional secretion pathways. While the mechanisms for sorting proteins and directing them to different vesicles are unknown, environmental stimuli is suggested to induce changes in components of extracellular vesicles [39]. It has been reported that environmental stimuli such as glucose concentration induce changes in S. cerevisiae extracellular vesicles [40], in particular, glucose starvation induces secretion of extracellular vesicles [41]. It has been reported that extracellular vesicles of $C$. neoformans stay in the cell wall [42]. Alternatively, it may be possible to block the secretion of specific extracellular vesicles by targeting molecules specific to the target vesicle, as suggested by Matos Baltazar and colleagues [43]. Taken together, extracellular vesicles are not uniform in their size, nor in their components and final destination.

\subsubsection{Unconventional Secretion of Acb1 and Sod1p in Fungal Cells}

Although unconventional secretory machineries are mostly unknown, the UPS pathways of several unconventionally secreted proteins are relatively well investigated. For example, the secretion of Acb1p is autophagosome-dependent [44] via an autophagosome-mediated cellular component [45] 
called a compartment for unconventional protein secretion (CUPS) [46]. Cytoplasmic Acb1p is recruited into CUPS by Grh1p, then compartmentalized into vesicles, and transported by endosomes. The endosome compartments are incorporated into a multivesicular body (MVB) by an endosomal sorting complex required for transport (ESCRT)-dependent processes. Then, MVB is fused with soluble $\mathrm{N}$-ethylmaleimide-sensitive factor attachment protein receptor (SNARE) proteins such as Sso1p [17].

The secreted Acb1 contributes to morphological changes in C. neoformans [47]. Recently, Sod1p was found to be secreted via the same machinery as Acb1p [48]. Interestingly, both Acb1p and Sod1p have Asp-Glu di-acidic motifs and substitution of these residues to alanine partially inhibits their secretion [48]. These findings suggest that there are protein domains or motifs that specifically promote unconventional secretion, many of which remain to be identified.

\subsubsection{Unconventional Secretion of Glycolytic Enzymes}

The glycolytic enzyme enolase is a protein for which the secretion pathway remains to be clarified. While enolase has been found in extracellular vesicles of C. albicans [11] and S. cerevisiae [31], the secretion of $S$. cerevisiae enolase, or Eno2p, is a SNARE protein Tlg2p-dependent, while it is autophagy-related protein- independent [14]. Moreover, several amino acid sequences or domains that are related to the unconventional secretion of enolase have been reported [14,49-51]. Some unconventionally secreted proteins are possibly secreted via more than one pathway [30], and this might be the case for enolase. It would be important to develop markers and/or mutant cell lines in which specific secretion pathways can be investigated.
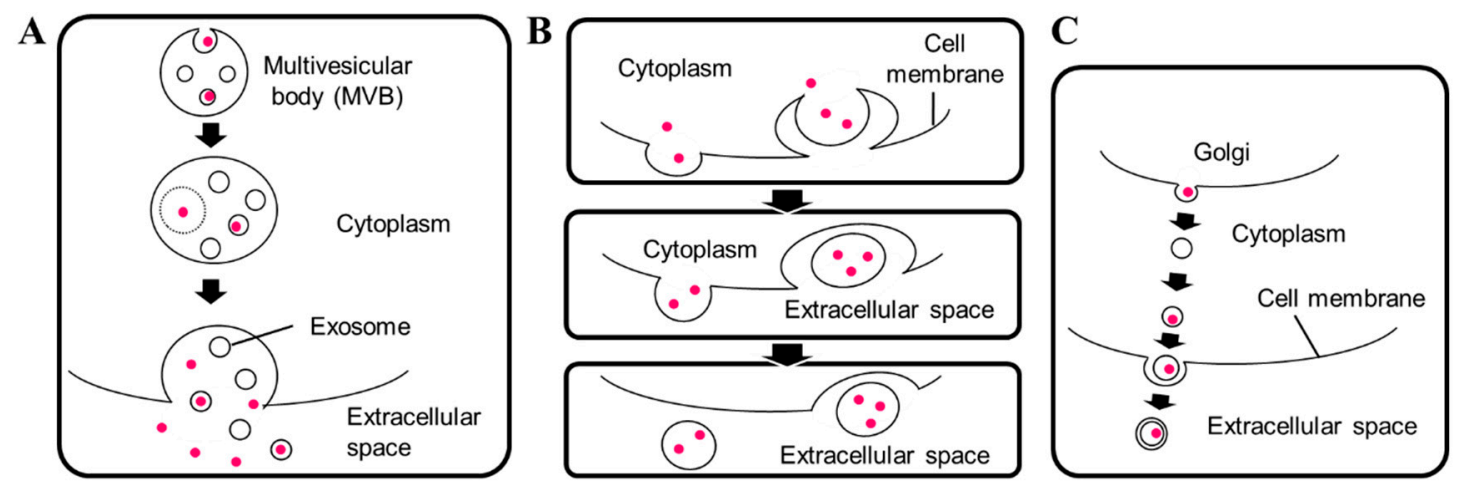

Figure 1. Different types of extracellular vesicle secretion in fungi. Prepared in reference with previous reports [25,52]. (A) Exosomes secreted via fusion of multivesicular body with plasma membranes; (B) microvesicles budding from cytoplasm; (C) Golgi-involved production of membrane vesicles. Pink dot: unconventionally secreted proteins.

\section{Approaches for Evaluating Unconventional Protein Secretion}

Fungal proteomics have identified various unconventionally secreted proteins, including some related to fungal allergens. While the presence of metabolic enzymes in the extracellular space can be caused by cell death or leakage, the presence of enolase among secreted proteins has been gradually accepted as not accidental [53].

Recently, Vivek-Ananth and colleagues performed in silico analysis on proteome datasets of opportunistic fungal pathogens, including Aspergillus fumigatus and ten other Aspergillus species. They developed a workflow to determine whether the proteins are secreted via conventional or unconventional secretion pathways. They estimated that in A. fumigatus, approximately $0.65 \%$ of the proteome is secreted via unconventional secretion and $6.1 \%$ of the proteome is secreted via conventional secretion, represented with 64 unconventionally secreted proteins and 598 conventionally secreted proteins, respectively [54]. 


\subsection{Methods of Secretome Analysis}

Several steps are required for identifying and evaluating UPS in fungal cells. Although fungi usually have a thick cell wall, intracellular proteins easily leak because of mechanical stress and cell death. In S. cerevisiae, the total number of protein molecules per cell is $\sim 5.0 \times 10^{7}$ [55], while the number of Eno2p molecules per cell is $\sim 9.3 \times 10^{5}$ [56], representing up to $2 \%$ of total proteins. Among other proteins, the numbers of actin (Act1p), Acb1p, Sod1p, and heat shock protein 70 (SSA1p) molecules per cell are $\sim 1.2 \times 10^{5}, \sim 1.4 \times 10^{5}, \sim 1.2 \times 10^{5}$, and $\sim 3.7 \times 10^{5}$, respectively [56]. Abundant proteins in the cell are detected easily in extracellular space when cell membranes are disturbed. Therefore, it is essential to construct methods for preparing samples carefully. Some validated unconventionally secreted proteins may be used as positive controls for unconventional secretion. On the other hand, green fluorescent proteins (GFP) and their derivatives are not secreted and could be used as negative controls $[14,57]$. It is essential to use these proteins for quality control when samples are prepared for tests [52]. The method below could be implemented to evaluate UPS:

1. Prepare extracellular protein samples

2. Test the samples by SDS-PAGE and Western blotting

3. Non-targeted proteome analysis of secreted proteins

4. Reconstruction of the UPS on specific proteins for further analysis

In the first step, extracellular proteins are prepared according to the purpose of research. Detailed methods for sample preparation can be found in previous reports [28,52]. Samples should be carefully prepared, avoiding unnecessary physical or chemical stresses to the cells. It is highly recommended, especially when the experiment is done for the first time, to use a proven intracellular protein such as GFP, as a standard to evaluate leakage.

In the second step, prepared proteins are tested by SDS-PAGE and Western blotting to validate contamination of leaked proteins into the samples. Antibodies against validated unconventionally secreted proteins, as well as negative controls, such as GFP, can be used.

In the third step, proteins are processed and detected by 2-DE followed by MS analysis, LC-MS/MS analysis, or other relevant methods. Since a number of proteome data are available, the beginning of this step can be substituted by in silico analysis of previous results, provided the methods used for protein preparation are carefully checked for each case. It also should be noted that it does not mean deposited proteins are secreted at any time. It has been reported that nutrient starvation changes the secretome of fungi [46]. In addition, proteomic approaches are based on the number of detectable proteins or peptide fragments, meaning that proteins with low abundance are likely not detected.

In the final step, unconventional secretion of specific proteins should be validated. Reconstruction of unconventional secretion using plasmids [52] would be effective to adjust protein levels in the cells, to validate secretion, and to determine the detailed secretion pathway of the target proteins.

\subsection{Proteome Databases}

Secretome data are usually deposited to proteome databases such as ProteomeXchange [58], which is a consortium of global proteome databases [59,60], including PRIDE [61,62], PeptideAtlas [63,64], MassIVE [65], jPOST [66,67], iProX [68], and Panorama [69,70]. Because of standardization of data formats and experimental workflows, the deposited data are becoming increasingly accessible and reusable [71]. Fungal secreted proteins can be found easily by species-specific searches of the database. Currently, since secreted proteins of pathogenic and allergenic fungi are often harmful to host cells, the importance of fungal secretome data is widely accepted [72]. This awareness possibly accelerates the deposition and utilization of proteome databases. 


\subsection{Re-Analysis of Fungal Secretome}

There are several ways of re-analyzing previous secretome data, including manual downloading and sorting of excel files submitted along with published reports. Since there are less reports for which the proteome data are submitted to proteome databases than there are with data attached as excel files or tables as shown in Table 1, manual treatment of the data as well as development of custom software is still effective. For the data available through the PRIDE database, due to recent updates, re-analysis of the data is becoming easier [73].

For re-analyzing proteome data deposited to PRIDE database, PRIDE Inspector [74,75] is used. After installation, PRIDE Inspector can be used on personal devices. By downloading the data after searching with ID as seen in Table 1, the data can be visualized on the software. However, it is still difficult to comparatively analyze results from different projects, because experimental conditions, methods for preparation, databases used for analysis, and instrumental settings vary dependent on each project. Still, since raw data is submitted to the database, re-analyzing several data on the same platform is becoming easier.

Table 2 shows the commonly detected extracellular proteins in four previous reports $[14,31,40,76]$. In addition to Acb1p and Sod1p discussed previously, the proteins listed are possibly unconventionally secreted proteins.

Table 1. Recent proteome data of extracellular proteins in some allergenic and/or pathogenic fungi.

\begin{tabular}{|c|c|c|c|c|c|c|}
\hline $\begin{array}{l}\text { Major Fungi with } \\
\text { Known Allergens }\end{array}$ & $\begin{array}{c}\text { Number of } \\
\text { Extracellular } \\
\text { Proteins Identified }\end{array}$ & Method $^{1}$ & Ref. & Year & Database $^{2}$ & ID \\
\hline \multirow{3}{*}{ Alternaria alternata } & 1383 & LC-MS/MS & [77] & 2016 & - & - \\
\hline & 1315 & iTRAQ & {$[78]$} & 2017 & - & - \\
\hline & 95 & $2-\mathrm{DE}$ & [79] & 2018 & - & - \\
\hline \multirow{3}{*}{$\begin{array}{l}\text { Aspergillus } \\
\text { fumigatus }\end{array}$} & 64 & 2-DE & {$[80]$} & 2011 & - & - \\
\hline & 128 & SDS-PAGE, LC-MS/MS & {$[81]$} & 2018 & - & - \\
\hline & 437 & $\begin{array}{l}\text { In sillico analysis of } \\
\text { previous secretome data }\end{array}$ & {$[54]$} & 2018 & - & - \\
\hline \multirow{2}{*}{ Beauveria bassiana } & 13 & 2-DE & {$[82]$} & 2007 & - & - \\
\hline & 50 & LC-MS/MS & [83] & 2016 & - & - \\
\hline \multirow{9}{*}{ Candida albicans } & 27 & 2-DE & {$[84]$} & 2002 & W2 & - \\
\hline & 14 & LC-MS/MS & {$[85]$} & 2004 & - & - \\
\hline & 48 & 2-DE & {$[86]$} & 2006 & - & - \\
\hline & 50 & LC-MS/MS & {$[87]$} & 2009 & - & - \\
\hline & 143 & LC-MS/MS & {$[88]$} & 2010 & - & - \\
\hline & 84 & LC-MS/MS & {$[89]$} & 2011 & - & - \\
\hline & 41 & LC-MS/MS & {$[90]$} & 2012 & PX & PXD000008 \\
\hline & 96 & LC-MS/MS & [11] & 2015 & $\mathrm{PX}$ & PXD000525 \\
\hline & 170 & LC-MS/MS & [91] & 2015 & $\mathrm{PX}$ & PXD000525 \\
\hline \multirow{7}{*}{$\begin{array}{l}\text { Saccharomyces } \\
\text { cerevisiae }\end{array}$} & 99 & 2-DE LC-MS/MS & [92] & 2010 & - & - \\
\hline & 219 & LC-MS/MS & [93] & 2010 & - & - \\
\hline & 127 & LC-MS/MS & {$[77]$} & 2010 & - & - \\
\hline & 42 & LC-MS/MS & [78] & 2011 & - & - \\
\hline & 42 & LC-MS & [79] & 2012 & $\mathrm{P}$ & PRD000729 \\
\hline & 347 & iTRAQ & [80] & 2014 & - & - \\
\hline & 694 & $\begin{array}{l}\text { 2-DE MALDI-TOF/TOF } \\
\text { LC-MS/MS }\end{array}$ & {$[81]$} & 2015 & PX & PXD001133 \\
\hline
\end{tabular}

Table 2. Extracellular proteins of S. cerevisiae commonly detected by four independent analysis $[14,31,40,76]$.

\begin{tabular}{|c|c|c|}
\hline Cellular Process & Description & Accession Number \\
\hline \multirow{6}{*}{ Carbohydrate Metabolism } & Cdc19p, Pyruvate kinase & gil 6319279 \\
\hline & Enolp. Enolase & gil 6321693 \\
\hline & Eno2p, Enolase II & gil 6321968 \\
\hline & Pdc1p, Major pyruvate decarboxylase & gil 6323073 \\
\hline & Pgk1p, 3-Phosphoglycerate kinase & gi | 10383781 \\
\hline & $\begin{array}{l}\text { Tdh3p, Glyceraldehyde-3-phosphate } \\
\text { dehydrogenase }\end{array}$ & gil 6321631 \\
\hline Protein Folding & Ssa1p, Hsp70 family & gi | 144228166 \\
\hline Other Functions & Tif2p, Translation initiation factor eIF4A & gi | 6322323 \\
\hline
\end{tabular}




\section{Unconventionally Secreted Fungal Allergens}

Most fungal allergens are extracellular proteins. Among previously reported fungal allergens, 18 do not contain known SPs used in the CPS pathway [6,7], shown in Table 3. Initially, these proteins without SPs were considered intracellular allergens [1]. Proteomic analyses detected these proteins in several fungal species as seen in Table 4 , and they are possibly unconventionally secreted. Among fungal allergens, superoxide dismutase (SOD) and enolase are the only unconventionally secreted proteins for which the secretion machineries are studied.

Table 3. Fungal allergens without known secretion signal sequences.

\begin{tabular}{|c|c|c|c|c|}
\hline Classification & Allergens & Species & $\begin{array}{c}\text { Genbank } \\
\text { Accession No. }\end{array}$ & Ref. \\
\hline \multirow{9}{*}{ Translation } & \multirow{3}{*}{ Acid ribosomal protein $\mathrm{P} 1$} & Alternaria alternata & X84216 & [95] \\
\hline & & Cladosporium herbarum & X85180 & {$[96]$} \\
\hline & & $\begin{array}{l}\text { Penicillium } \\
\text { brevicompactum }\end{array}$ & AY786077 & [97] \\
\hline & \multirow{4}{*}{ Acid ribosomal protein P2 } & Alternaria alternata & X78222, U87806 & [95] \\
\hline & & Aspergillus fumigatus & AJ224333 & [98] \\
\hline & & Cladosporium herbarum & X78223 & {$[95,99]$} \\
\hline & & Fusarium culmorum & AY077706 & [100] \\
\hline & L3 ribosomal protein & Aspergillus fumigatus & AF464911 & [101] \\
\hline & Elongation factor 1 beta & Penicillium citrinum & AY363911 & [102] \\
\hline \multirow{17}{*}{ Metabolism } & Alcohol dehydrogenase & Candida albicans & X81694 & [103] \\
\hline & & Alternaria alternata & X78227, P42041 & [95] \\
\hline & Aldehyde dehydrogenase & Beauveria bassiana & DQ767721 & [104] \\
\hline & & Cladosporium herbarum & X78228 & [95] \\
\hline & \multirow{9}{*}{ Enolase } & Alternaria alternata & U82437 & [105] \\
\hline & & Aspergillus fumigatus & AF284645 & [106] \\
\hline & & Beauveria bassiana & DQ767719 & [104] \\
\hline & & Candida albicans & L04943 & {$[107,108]$} \\
\hline & & Cladosporium herbarum & X78226 & [95] \\
\hline & & Curoularia lunata & AY034826 & [109] \\
\hline & & Penicillium citrinum & AF254643 & [106] \\
\hline & & Saccharomyces cerevisiae & J01322 & {$[106,110]$} \\
\hline & & Rhodotorula mucilaginosa & AY547285 & [111] \\
\hline & Formate dehydrogenase & Candida boidinii & AJ011046 & [112] \\
\hline & \multirow{2}{*}{ Mannitol dehydrogenase } & Alternaria alternata & AY191815 & [113] \\
\hline & & Cladosporium herbarum & AY191816 & [114] \\
\hline & $\begin{array}{l}\text { Mitochondrial malate } \\
\text { dehydrogenase }\end{array}$ & Malassezia furfur & AF084828 & [115] \\
\hline \multirow{5}{*}{$\begin{array}{l}\text { Heat shock } \\
\text { proteins }\end{array}$} & \multirow{3}{*}{ HSP70 } & Alternaria alternata & U87807, U87808 & [116] \\
\hline & & Cladosporium herbarum & X81860 & [117] \\
\hline & & Penicillium citrinum & U64207 & [118] \\
\hline & HSP88 & Malassezia sympodialis & AJ428052 & {$[119,120]$} \\
\hline & HSP90 & Aspergillus fumigatus & U92465 & [121] \\
\hline \multirow{7}{*}{ Others } & \multirow{3}{*}{ MnSOD } & Aspergillus fumigatus & U53561 & [122] \\
\hline & & Saccharomyces cerevisiae & X02156 & [123] \\
\hline & & Malassezia sympodialis & AJ548421 & [120] \\
\hline & Peptidyl-prolyl isomerase & Aspergillus fumigatus & AJ006689 & [124] \\
\hline & $\begin{array}{l}\text { Protein disulfide } \\
\text { isomerase }\end{array}$ & Alternaria alternata & X84217 & [95] \\
\hline & Thioredoxin-like protein & Fusarium culmorum & AY077707 & [100] \\
\hline & GST & Alternaria alternata & AY514673 & [125] \\
\hline
\end{tabular}

Prepared with reference to previous reports [1,126]. Absence of secretion signal peptide was determined by SignalP 4.1 [127]. HSP: heat shock proteins, SOD: superoxide dismutase, GST: Glutathione S-transferase. 
Table 4. Fungal allergen-related proteins detected by secretome analysis.

\begin{tabular}{|c|c|c|c|}
\hline Classification & Secreted Protein & Species & Ref. \\
\hline \multirow{5}{*}{ Metabolism } & Alcohol dehydrogenase & $\begin{array}{c}\text { Alternaria alternata } \\
\text { Candida albicans } \\
\text { Saccharomyces cerevisiae }\end{array}$ & $\begin{array}{c}{[78]} \\
{[84,91]} \\
{[31,40,76]}\end{array}$ \\
\hline & $\begin{array}{c}\text { Aldehyde } \\
\text { dehydrogenase }\end{array}$ & $\begin{array}{c}\text { Candida albicans } \\
\text { Saccharomyces cerevisiae }\end{array}$ & $\begin{array}{c}{[91]} \\
{[31,40]}\end{array}$ \\
\hline & Enolase & $\begin{array}{l}\text { Aspergillus fumigatus } \\
\text { Candida albicans } \\
\text { Saccharomyces cerevisiae }\end{array}$ & $\begin{array}{c}{[80]} \\
{[11,84,89,91]} \\
{[14,31,40,76,93]}\end{array}$ \\
\hline & Formate dehydrogenase & $\begin{array}{l}\text { Aspergillus fumigatus } \\
\text { Candida albicans }\end{array}$ & $\begin{array}{l}{[80]} \\
{[91]}\end{array}$ \\
\hline & $\begin{array}{l}\text { Mitochondrial malate } \\
\text { dehydrogenase }\end{array}$ & $\begin{array}{l}\text { Aspergillus fumigatus } \\
\text { Saccharomyces cerevisiae }\end{array}$ & $\begin{array}{c}{[80]} \\
{[14,40]}\end{array}$ \\
\hline \multirow{2}{*}{ Heat shock proteins } & HSP70 & $\begin{array}{c}\text { Candida albicans } \\
\text { Saccharomyces cerevisiae }\end{array}$ & $\begin{array}{l}{[11,91]} \\
{[31,40]} \\
\end{array}$ \\
\hline & HSP90 & $\begin{array}{c}\text { Candida albicans } \\
\text { Saccharomyces cerevisiae }\end{array}$ & $\begin{array}{c}{[84,89]} \\
{[14,31,40]}\end{array}$ \\
\hline \multirow{5}{*}{ Others } & SOD & $\begin{array}{l}\text { Aspergillus fumigatus } \\
\text { Saccharomyces cerevisiae }\end{array}$ & $\begin{array}{c}{[80]} \\
{[14,31,40,76]}\end{array}$ \\
\hline & $\begin{array}{l}\text { Peptidyl-prolyl } \\
\text { isomerase }\end{array}$ & $\begin{array}{c}\text { Candida albicans } \\
\text { Saccharomyces cerevisiae }\end{array}$ & $\begin{array}{c}{[91]} \\
{[14,31,40]}\end{array}$ \\
\hline & $\begin{array}{l}\text { Protein disulfide } \\
\text { isomerase }\end{array}$ & $\begin{array}{c}\text { Candida albicans } \\
\text { Saccharomyces cerevisiae }\end{array}$ & $\begin{array}{c}{[88]} \\
{[14,31]}\end{array}$ \\
\hline & Thioredoxin & $\begin{array}{c}\text { Candida albicans } \\
\text { Saccharomyces cerevisiae }\end{array}$ & $\begin{array}{c}{[91]} \\
{[14,31,40]} \\
\end{array}$ \\
\hline & GST & Saccharomyces cerevisiae & {$[31]$} \\
\hline
\end{tabular}

HSP: heat shock proteins, SOD: superoxide dismutase, GST: Glutathione S-transferase.

\section{Discussion and Future Perspectives}

Fungal extracellular vesicles as vectors for unconventionally secreted proteins attract increased attention because of their potential roles in host-microbe interactions [32,34,39]. Since secretome analysis in addition to genome editing of fungal cells are becoming popular, efforts to regulate fungal secretome [128] would be accelerated. In terms of fungal extracellular proteins as allergens, the importance of extracellular vesicles as cell wall components should be noted [39], since vesicles on the cell wall allow exposure of host cells with concentrated proteins when the vesicle is ruptured.

Notably, most of the unconventionally secreted proteins related to fungal allergens are also reported in S. cerevisiae cells, as seen in Table 4. S. cerevisiae is a model organism extensively studied for understanding the conventional secretion pathway, and mutants in regulatory molecules important for conventional secretion are easily available [129-132]. With genome-wide knockout [133,134], overexpression [135], and knockdown [135,136] libraries, S. cerevisiae remains an attractive model for investigating unconventional secretion pathway of proteins, including fungal allergens $[14,52,137]$.

The use of extracellular vesicles for therapeutics [138] is a possible way of utilizing fungal extracellular vesicles. For the purpose, it would be important to increase the number of extracellular vesicles secreted by fungi, as unconventional secretion is not a major secretory pathway in fungal cells [54].

Fungal unconventional secretion has been driven by the development of proteome analysis [10,11]. Consequent development of the database and related tools [59] now assists the comparative systems analysis of numerous secretome data as reported recently [54]. Accumulating further data would enable classification of unconventional secretion pathways, with the development of mutant strains in which 
a specific pathway is deficient. These developments would clarify species-dependent unconventional secretion pathways, providing insights into strategies of pathogenic fungi in the future.

Author Contributions: Conceptualization, N.M. and M.U.; Writing-Original Draft Preparation, N.M.; Writing-Review \& Editing, N.M. and M.U.; Visualization, N.M.; Project Administration, N.M. and M.U.; Funding Acquisition, M.U.

Funding: This work was partly supported by JST CREST (Grant No. JPMJCR16G2).

Conflicts of Interest: The authors declare no conflicts of interest.

\section{References}

1. Simon-Nobbe, B.; Denk, U.; Poll, V.; Rid, R.; Breitenbach, M. The spectrum of fungal allergy. Int. Arch. Allergy Immunol. 2008, 145, 58-86. [CrossRef] [PubMed]

2. Falahati, M.; Ghanbari, S.; Ebrahimi, M.; Ghazanfari, M.; Bazrafshan, F.; Farahyar, S.; Falak, R. Fractionation and identification of the allergic proteins in Aspergillus species. Curr. Med. Mycol. 2016, 2, 37-45. [CrossRef] [PubMed]

3. Asif, A.R.; Oellerich, M.; Amstrong, V.W.; Riemenschneider, B.; Monod, M.; Reichard, U. Proteome of conidial surface associated proteins of Aspergillus fumigatus reflecting potential vaccine candidates and allergens. J. Proteome Res. 2006, 5, 954-962. [CrossRef] [PubMed]

4. Chaffin, W.L.; Lopez-Ribot, J.L.; Casanova, M.; Gozalbo, D.; Martinez, J.P. Cell wall and secreted proteins of Candida albicans: Identification, function, and expression. Microbiol. Mol. Biol. Rev. 1998, 62, $130-180$. [PubMed]

5. Schneiter, R.; Di Pietro, A. The cap protein superfamily: Function in sterol export and fungal virulence. Biomol. Concepts 2013, 4, 519-525. [CrossRef] [PubMed]

6. Ferro-Novick, S.; Brose, N. Nobel 2013 physiology or medicine: Traffic control system within cells. Nature 2013, 504, 98. [CrossRef] [PubMed]

7. Palade, G. Intracellular aspects of the process of protein synthesis. Science 1975, 189, 867. [CrossRef] [PubMed]

8. Lopez-Villar, E.; Monteoliva, L.; Larsen, M.R.; Sachon, E.; Shabaz, M.; Pardo, M.; Pla, J.; Gil, C.; Roepstorff, P.; Nombela, C. Genetic and proteomic evidences support the localization of yeast enolase in the cell surface. Proteomics 2006, 6 (Suppl. 1), S107-S118. [CrossRef] [PubMed]

9. Rodrigues, M.L.; Nakayasu, E.S.; Almeida, I.C.; Nimrichter, L. The impact of proteomics on the understanding of functions and biogenesis of fungal extracellular vesicles. J. Proteom. 2014, 97, 177-186. [CrossRef] [PubMed]

10. Faria-Oliveira, F.; Carvalho, J.; Ferreira, C.; Hernaez, M.L.; Gil, C.; Lucas, C. Quantitative differential proteomics of yeast extracellular matrix: There is more to it than meets the eye. BMC Microbiol. 2015, 15, 271. [CrossRef] [PubMed]

11. Gil-Bona, A.; Llama-Palacios, A.; Parra, C.M.; Vivanco, F.; Nombela, C.; Monteoliva, L.; Gil, C. Proteomics unravels extracellular vesicles as carriers of classical cytoplasmic proteins in Candida albicans. J. Proteome Res. 2015, 14, 142-153. [CrossRef] [PubMed]

12. Wang, W.; Jeffery, C.J. An analysis of surface proteomics results reveals novel candidates for intracellular/surface moonlighting proteins in bacteria. Mol. Biosyst. 2016, 12, 1420-1431. [CrossRef] [PubMed]

13. Gil-Bona, A.; Amador-Garcia, A.; Gil, C.; Monteoliva, L. The external face of Candida albicans: A proteomic view of the cell surface and the extracellular environment. J. Proteom. 2018, 180, 70-79. [CrossRef] [PubMed]

14. Miura, N.; Kirino, A.; Endo, S.; Morisaka, H.; Kuroda, K.; Takagi, M.; Ueda, M. Tracing putative trafficking of the glycolytic enzyme enolase via SNARE-driven unconventional secretion. Eukaryot Cell 2012, 11, 1075-1082. [CrossRef] [PubMed]

15. Conesa, A.; Punt, P.J.; van Luijk, N.; van den Hondel, C.A. The secretion pathway in filamentous fungi: A biotechnological view. Fungal Genet. Biol. 2001, 33, 155-171. [CrossRef] [PubMed]

16. Feizi, A.; Osterlund, T.; Petranovic, D.; Bordel, S.; Nielsen, J. Genome-scale modeling of the protein secretory machinery in yeast. PLoS ONE 2013, 8, e63284. [CrossRef] [PubMed]

17. Malhotra, V. Unconventional protein secretion: An evolving mechanism. EMBO J. 2013, 32, 1660-1664. [CrossRef] [PubMed] 
18. DTU Bioinformatics Department of Bio and Health Informatics ServerIP 4.1 Server. Available online: http:/ / www.cbs.dtu.dk/services/SignalP (accessed on 27 August 2018).

19. Nielsen, H. Predicting secretory proteins with SignalP. Methods Mol. Biol. 2017, 1611, 59-73. [PubMed]

20. PSORT: Prediction of Protein Sorting Signals and Localization Sites in Amino Acid Sequences. Available online: https:/ / psort.hgc.jp (accessed on 27 August 2018).

21. Nakai, K.; Horton, P. PSort: A program for detecting sorting signals in proteins and predicting their subcellular localization. Trends Biochem. Sci. 1999, 24, 34-36. [CrossRef]

22. DTU Bioinformatics Department of Bio and Health Informatics TargetP 1.1 Server. Available online: http: / / www.cbs.dtu.dk/services/TargetP (accessed on 27 August 2018).

23. Emanuelsson, O.; Brunak, S.; von Heijne, G.; Nielsen, H. Locating proteins in the cell using TargetP, SignalP and related tools. Nat. Protoc. 2007, 2, 953-971. [CrossRef] [PubMed]

24. Sperschneider, J.; Williams, A.H.; Hane, J.K.; Singh, K.B.; Taylor, J.M. Evaluation of secretion prediction highlights differing approaches needed for oomycete and fungal effectors. Front. Plant Sci. 2015, 6, 1168. [CrossRef] [PubMed]

25. Wolf, J.M.; Casadevall, A. Challenges posed by extracellular vesicles from eukaryotic microbes. Curr. Opin Microbiol. 2014, 22, 73-78. [CrossRef] [PubMed]

26. Robbertse, B.; Tatusova, T. Fungal genome resources at NCBI. Mycology 2011, 2, 142-160. [PubMed]

27. Colombo, M.; Raposo, G.; Thery, C. Biogenesis, secretion, and intercellular interactions of exosomes and other extracellular vesicles. Annu. Rev. Cell Dev. Biol. 2014, 30, 255-289. [CrossRef] [PubMed]

28. Rodrigues, M.L.; Oliveira, D.L.; Vargas, G.; Girard-Dias, W.; Franzen, A.J.; Frases, S.; Miranda, K.; Nimrichter, L. Analysis of yeast extracellular vesicles. Methods Mol. Biol. 2016, 1459, 175-190. [PubMed]

29. Rodrigues, M.L.; Nakayasu, E.S.; Oliveira, D.L.; Nimrichter, L.; Nosanchuk, J.D.; Almeida, I.C.; Casadevall, A. Extracellular vesicles produced by Cryptococcus neoformans contain protein components associated with virulence. Eukaryot Cell 2008, 7, 58-67. [CrossRef] [PubMed]

30. Vallejo, M.C.; Nakayasu, E.S.; Matsuo, A.L.; Sobreira, T.J.; Longo, L.V.; Ganiko, L.; Almeida, I.C.; Puccia, R. Vesicle and vesicle-free extracellular proteome of Paracoccidioides brasiliensis: Comparative analysis with other pathogenic fungi. J. Proteome Res. 2012, 11, 1676-1685. [CrossRef] [PubMed]

31. Oliveira, D.L.; Nakayasu, E.S.; Joffe, L.S.; Guimaraes, A.J.; Sobreira, T.J.; Nosanchuk, J.D.; Cordero, R.J.; Frases, S.; Casadevall, A.; Almeida, I.C.; et al. Characterization of yeast extracellular vesicles: Evidence for the participation of different pathways of cellular traffic in vesicle biogenesis. PLoS ONE 2010, 5, e11113. [CrossRef] [PubMed]

32. Zamith-Miranda, D.; Nimrichter, L.; Rodrigues, M.L.; Nosanchuk, J.D. Fungal extracellular vesicles: Modulating host-pathogen interactions by both the fungus and the host. Microbes Infect 2018. [CrossRef] [PubMed]

33. Joffe, L.S.; Nimrichter, L.; Rodrigues, M.L.; Del Poeta, M. Potential roles of fungal extracellular vesicles during infection. mSphere 2016, 1. [CrossRef] [PubMed]

34. Johansson, H.J.; Vallhov, H.; Holm, T.; Gehrmann, U.; Andersson, A.; Johansson, C.; Blom, H.; Carroni, M.; Lehtio, J.; Scheynius, A. Extracellular nanovesicles released from the commensal yeast Malassezia sympodialis are enriched in allergens and interact with cells in human skin. Sci. Rep. 2018, 8, 9182. [CrossRef] [PubMed]

35. Kabani, M.; Melki, R. Sup35p in its soluble and prion states is packaged inside extracellular vesicles. MBio 2015, 6. [CrossRef] [PubMed]

36. Kreimer, S.; Belov, A.M.; Ghiran, I.; Murthy, S.K.; Frank, D.A.; Ivanov, A.R. Mass-spectrometry-based molecular characterization of extracellular vesicles: Lipidomics and proteomics. J. Proteome Res. 2015, 14, 2367-2384. [CrossRef] [PubMed]

37. Rodrigues, M.L.; Franzen, A.J.; Nimrichter, L.; Miranda, K. Vesicular mechanisms of traffic of fungal molecules to the extracellular space. Curr. Opin. Microbiol. 2013, 16, 414-420. [CrossRef] [PubMed]

38. Rodrigues, M.L.; Godinho, R.M.; Zamith-Miranda, D.; Nimrichter, L. Traveling into outer space: Unanswered questions about fungal extracellular vesicles. PLoS Pathog 2015, 11, e1005240. [CrossRef] [PubMed]

39. Nimrichter, L.; de Souza, M.M.; Del Poeta, M.; Nosanchuk, J.D.; Joffe, L.; Tavares Pde, M.; Rodrigues, M.L. Extracellular vesicle-associated transitory cell wall components and their impact on the interaction of fungi with host cells. Front. Microbiol. 2016, 7, 1034. [CrossRef] [PubMed]

40. Giardina, B.J.; Stanley, B.A.; Chiang, H.L. Glucose induces rapid changes in the secretome of Saccharomyces cerevisiae. Proteome Sci. 2014, 12, 9. [CrossRef] [PubMed] 
41. Stein, K.; Chiang, H.L. Exocytosis and endocytosis of small vesicles across the plasma membrane in Saccharomyces cerevisiae. Membranes 2014, 4, 608-629. [CrossRef] [PubMed]

42. Wolf, J.M.; Espadas-Moreno, J.; Luque-Garcia, J.L.; Casadevall, A. Interaction of Cryptococcus neoformans extracellular vesicles with the cell wall. Eukaryot Cell 2014, 13, 1484-1493. [CrossRef] [PubMed]

43. Matos Baltazar, L.; Nakayasu, E.S.; Sobreira, T.J.; Choi, H.; Casadevall, A.; Nimrichter, L.; Nosanchuk, J.D. Antibody binding alters the characteristics and contents of extracellular vesicles released by Histoplasma capsulatum. mSphere 2016, 1. [CrossRef] [PubMed]

44. Duran, J.M.; Anjard, C.; Stefan, C.; Loomis, W.F.; Malhotra, V. Unconventional secretion of Acb1 is mediated by autophagosomes. J. Cell Biol. 2010, 188, 527-536. [CrossRef] [PubMed]

45. Bruns, C.; McCaffery, J.M.; Curwin, A.J.; Duran, J.M.; Malhotra, V. Biogenesis of a novel compartment for autophagosome-mediated unconventional protein secretion. J. Cell Biol. 2011, 195, 979-992. [CrossRef] [PubMed]

46. Cruz-Garcia, D.; Malhotra, V.; Curwin, A.J. Unconventional protein secretion triggered by nutrient starvation. Semin. Cell Dev. Biol. 2018. [CrossRef] [PubMed]

47. Xu, X.; Zhao, Y.; Kirkman, E.; Lin, X. Secreted Acb1 contributes to the yeast-to-hypha transition in Cryptococcus neoformans. Appl. Environ. Microbiol. 2016, 82, 1069-1079. [CrossRef] [PubMed]

48. Cruz-Garcia, D.; Brouwers, N.; Duran, J.M.; Mora, G.; Curwin, A.J.; Malhotra, V. A diacidic motif determines unconventional secretion of wild-type and ALS-linked mutant SOD1. J. Cell Biol. 2017. [CrossRef] [PubMed]

49. Yang, C.K.; Zhang, X.Z.; Lu, C.D.; Tai, P.C. An internal hydrophobic helical domain of Bacillus subtilis enolase is essential but not sufficient as a non-cleavable signal for its secretion. Biochem. Biophys. Res. Commun. 2014, 446, 901-905. [CrossRef] [PubMed]

50. Boel, G.; Pichereau, V.; Mijakovic, I.; Maze, A.; Poncet, S.; Gillet, S.; Giard, J.C.; Hartke, A.; Auffray, Y.; Deutscher, J. Is 2-phosphoglycerate-dependent automodification of bacterial enolases implicated in their export? J. Mol. Biol. 2004, 337, 485-496. [CrossRef] [PubMed]

51. Yang, C.K.; Ewis, H.E.; Zhang, X.; Lu, C.D.; Hu, H.J.; Pan, Y.; Abdelal, A.T.; Tai, P.C. Nonclassical protein secretion by Bacillus subtilis in the stationary phase is not due to cell lysis. J. Bacteriol. 2011, 193, 5607-5615. [CrossRef] [PubMed]

52. Miura, N.; Ueda, M. Evaluation of unconventional protein secretion in Saccharomyces cerevisiae. Methods Mol. Biol. 2015, 1270, 51-70. [PubMed]

53. Brown, K.J.; Formolo, C.A.; Seol, H.; Marathi, R.L.; Duguez, S.; An, E.; Pillai, D.; Nazarian, J.; Rood, B.R.; Hathout, Y. Advances in the proteomic investigation of the cell secretome. Expert Rev. Proteom. 2012, 9, 337-345. [CrossRef] [PubMed]

54. Vivek-Ananth, R.P.; Mohanraj, K.; Vandanashree, M.; Jhingran, A.; Craig, J.P.; Samal, A. Comparative systems analysis of the secretome of the opportunistic pathogen Aspergillus fumigatus and other Aspergillus species. Sci. Rep. 2018, 8, 6617. [CrossRef] [PubMed]

55. Futcher, B.; Latter, G.I.; Monardo, P.; McLaughlin, C.S.; Garrels, J.I. A sampling of the yeast proteome. Mol. Cell Biol. 1999, 19, 7357-7368. [CrossRef] [PubMed]

56. Kulak, N.A.; Pichler, G.; Paron, I.; Nagaraj, N.; Mann, M. Minimal, encapsulated proteomic-sample processing applied to copy-number estimation in eukaryotic cells. Nat. Methods 2014, 11, 319-324. [CrossRef] [PubMed]

57. Morisaka, H.; Matsui, K.; Tatsukami, Y.; Kuroda, K.; Miyake, H.; Tamaru, Y.; Ueda, M. Profile of native cellulosomal proteins of Clostridium cellulovorans adapted to various carbon sources. AMB Express 2012, 2, 37. [CrossRef] [PubMed]

58. ProteomeXchange. Available online: http://www.proteomexchange.org (accessed on 27 August 2018).

59. Deutsch, E.W.; Csordas, A.; Sun, Z.; Jarnuczak, A.; Perez-Riverol, Y.; Ternent, T.; Campbell, D.S.; Bernal-Llinares, M.; Okuda, S.; Kawano, S.; et al. The proteomeXchange consortium in 2017: Supporting the cultural change in proteomics public data deposition. Nucleic Acids Res. 2017, 45, D1100-D1106. [CrossRef] [PubMed]

60. Vizcaino, J.A.; Deutsch, E.W.; Wang, R.; Csordas, A.; Reisinger, F.; Rios, D.; Dianes, J.A.; Sun, Z.; Farrah, T.; Bandeira, N.; et al. ProteomeXchange provides globally coordinated proteomics data submission and dissemination. Nat. Biotechnol. 2014, 32, 223-226. [CrossRef] [PubMed]

61. PRIDE Archive. Available online: https://www.ebi.ac.uk/pride/archive (accessed on 27 August 2018). 
62. Vizcaino, J.A.; Csordas, A.; Del-Toro, N.; Dianes, J.A.; Griss, J.; Lavidas, I.; Mayer, G.; Perez-Riverol, Y.; Reisinger, F.; Ternent, T.; et al. 2016 update of the PRIDE database and its related tools. Nucleic Acids Res. 2016, 44, 11033. [CrossRef] [PubMed]

63. Peptide Atlas. Available online: http:/ / www.peptideatlas.org (accessed on 27 August 2018).

64. Deutsch, E.W. The PeptideAtlas project. Methods Mol. Biol. 2010, 604, 285-296. [PubMed]

65. Mass Spectrometry Interactive Virtual Environment MassIVE. Available online: https:/ / massive.ucsd.edu/ ProteoSAFe/static/massive.jsp (accessed on 27 August 2018).

66. Japan Proteome Standard Repository/Database jPOST. Available online: https://jpostdb.org (accessed on 27 August 2018).

67. Okuda, S.; Watanabe, Y.; Moriya, Y.; Kawano, S.; Yamamoto, T.; Matsumoto, M.; Takami, T.; Kobayashi, D.; Araki, N.; Yoshizawa, A.C.; et al. jPOSTrepo: An international standard data repository for proteomes. Nucleic Acids Res. 2017, 45, D1107-D1111. [CrossRef] [PubMed]

68. iProX-integrated Proteome resources. Available online: http:/ /www.iprox.org (accessed on 27 August 2018).

69. PanoramaWeb. Available online: https://panoramaweb.org/project/home/begin.view (accessed on 27 August 2018).

70. Sharma, V.; Eckels, J.; Taylor, G.K.; Shulman, N.J.; Stergachis, A.B.; Joyner, S.A.; Yan, P.; Whiteaker, J.R.; Halusa, G.N.; Schilling, B.; et al. Panorama: A targeted proteomics knowledge base. J. Proteome Res. 2014, 13, 4205-4210. [CrossRef] [PubMed]

71. Perez-Riverol, Y.; Alpi, E.; Wang, R.; Hermjakob, H.; Vizcaino, J.A. Making proteomics data accessible and reusable: Current state of proteomics databases and repositories. Proteomics 2015, 15, 930-949. [CrossRef] [PubMed]

72. Girard, V.; Dieryckx, C.; Job, C.; Job, D. Secretomes: The fungal strike force. Proteomics 2013, 13, 597-608. [CrossRef] [PubMed]

73. Vaudel, M.; Burkhart, J.M.; Zahedi, R.P.; Oveland, E.; Berven, F.S.; Sickmann, A.; Martens, L.; Barsnes, H. PeptideShaker enables reanalysis of MS-derived proteomics data sets. Nat. Biotechnol. 2015, 33, $22-24$. [CrossRef] [PubMed]

74. PRIDE Inspector. Available online: https://github.com/PRIDE-Toolsuite/pride-inspector (accessed on 27 August 2018).

75. Wang, R.; Fabregat, A.; Rios, D.; Ovelleiro, D.; Foster, J.M.; Cote, R.G.; Griss, J.; Csordas, A.; Perez-Riverol, Y.; Reisinger, F.; et al. PRIDE Inspector: A tool to visualize and validate ms proteomics data. Nat. Biotechnol. 2012, 30, 135-137. [CrossRef] [PubMed]

76. Braconi, D.; Amato, L.; Bernardini, G.; Arena, S.; Orlandini, M.; Scaloni, A.; Santucci, A. Surfome analysis of a wild-type wine Saccharomyces cerevisiae strain. Food Microbiol. 2011, 28, 1220-1230. [CrossRef] [PubMed]

77. Zeiner, C.A.; Purvine, S.O.; Zink, E.M.; Pasa-Tolic, L.; Chaput, D.L.; Haridas, S.; Wu, S.; LaButti, K.; Grigoriev, I.V.; Henrissat, B.; et al. Comparative analysis of secretome profiles of manganese(ii)-oxidizing Ascomycete fungi. PLoS ONE 2016, 11, e0157844. [CrossRef] [PubMed]

78. Zeiner, C.A.; Purvine, S.O.; Zink, E.M.; Pasa-Tolic, L.; Chaput, D.L.; Wu, S.; Santelli, C.M.; Hansel, C.M. Quantitative itraq-based secretome analysis reveals species-specific and temporal shifts in carbon utilization strategies among manganese(ii)-oxidizing Ascomycete fungi. Fungal Genet. Biol. 2017, 106, 61-75. [CrossRef] [PubMed]

79. Garcia-Calvo, L.; Ullan, R.V.; Fernandez-Aguado, M.; Garcia-Lino, A.M.; Balana-Fouce, R.; Barreiro, C. Secreted protein extract analyses present the plant pathogen Alternaria alternata as a suitable industrial enzyme toolbox. J. Proteom. 2018, 177, 48-64. [CrossRef] [PubMed]

80. Wartenberg, D.; Lapp, K.; Jacobsen, I.D.; Dahse, H.M.; Kniemeyer, O.; Heinekamp, T.; Brakhage, A.A. Secretome analysis of Aspergillus fumigatus reveals Asp-hemolysin as a major secreted protein. Int. J. Med. Microbiol. 2011, 301, 602-611. [CrossRef] [PubMed]

81. de Gouvea, P.F.; Bernardi, A.V.; Gerolamo, L.E.; de Souza Santos, E.; Riano-Pachon, D.M.; Uyemura, S.A.; Dinamarco, T.M. Transcriptome and secretome analysis of Aspergillus fumigatus in the presence of sugarcane bagasse. BMC Genom. 2018, 19, 232. [CrossRef] [PubMed]

82. Murad, A.M.; Laumann, R.A.; Mehta, A.; Noronha, E.F.; Franco, O.L. Screening and secretomic analysis of enthomopatogenic Beauveria bassiana isolates in response to cowpea weevil (Callosobruchus maculatus) exoskeleton. Comp. Biochem. Physiol. C Toxicol. Pharmacol. 2007, 145, 333-338. [CrossRef] [PubMed] 
83. Dionisio, G.; Kryger, P.; Steenberg, T. Label-free differential proteomics and quantification of exoenzymes from isolates of the entomopathogenic fungus Beauveria bassiana. Insects 2016, 7. [CrossRef] [PubMed]

84. Pitarch, A.; Sanchez, M.; Nombela, C.; Gil, C. Sequential fractionation and two-dimensional gel analysis unravels the complexity of the dimorphic fungus Candida albicans cell wall proteome. Mol. Cell Proteom. 2002, 1, 967-982. [CrossRef]

85. de Groot, P.W.; de Boer, A.D.; Cunningham, J.; Dekker, H.L.; de Jong, L.; Hellingwerf, K.J.; de Koster, C.; Klis, F.M. Proteomic analysis of Candida albicans cell walls reveals covalently bound carbohydrate-active enzymes and adhesins. Eukaryot Cell 2004, 3, 955-965. [CrossRef] [PubMed]

86. Thomas, D.P.; Bachmann, S.P.; Lopez-Ribot, J.L. Proteomics for the analysis of the Candida albicans biofilm lifestyle. Proteomics 2006, 6, 5795-5804. [CrossRef] [PubMed]

87. Maddi, A.; Bowman, S.M.; Free, S.J. Trifluoromethanesulfonic acid-based proteomic analysis of cell wall and secreted proteins of the Ascomycetous fungi Neurospora crassa and Candida albicans. Fungal Genet. Biol. 2009, 46, 768-781. [CrossRef] [PubMed]

88. Sorgo, A.G.; Heilmann, C.J.; Dekker, H.L.; Brul, S.; de Koster, C.G.; Klis, F.M. Mass spectrometric analysis of the secretome of Candida albicans. Yeast 2010, 27, 661-672. [CrossRef] [PubMed]

89. Sorgo, A.G.; Heilmann, C.J.; Dekker, H.L.; Bekker, M.; Brul, S.; de Koster, C.G.; de Koning, L.J.; Klis, F.M. Effects of fluconazole on the secretome, the wall proteome, and wall integrity of the clinical fungus Candida albicans. Eukaryot Cell 2011, 10, 1071-1081. [CrossRef] [PubMed]

90. Ene, I.V.; Heilmann, C.J.; Sorgo, A.G.; Walker, L.A.; de Koster, C.G.; Munro, C.A.; Klis, F.M.; Brown, A.J. Carbon source-induced reprogramming of the cell wall proteome and secretome modulates the adherence and drug resistance of the fungal pathogen Candida albicans. Proteomics 2012, 12, 3164-3179. [CrossRef] [PubMed]

91. Gil-Bona, A.; Monteoliva, L.; Gil, C. Global proteomic profiling of the secretome of Candida albicans ecm33 cell wall mutant reveals the involvement of ecm33 in sap2 secretion. J. Proteome Res. 2015, 14, 4270-4281. [CrossRef] [PubMed]

92. Insenser, M.R.; Hernaez, M.L.; Nombela, C.; Molina, M.; Molero, G.; Gil, C. Gel and gel-free proteomics to identify Saccharomyces cerevisiae cell surface proteins. J. Proteom. 2010, 73, 1183-1195. [CrossRef] [PubMed]

93. Rowe, J.D.; Harbertson, J.F.; Osborne, J.P.; Freitag, M.; Lim, J.; Bakalinsky, A.T. Systematic identification of yeast proteins extracted into model wine during aging on the yeast lees. J. Agric. Food Chem. 2010, 58, 2337-2346. [CrossRef] [PubMed]

94. World-2DPAGE List. Available online: https://world-2dpage.expasy.org/list (accessed on 27 August 2018).

95. Achatz, G.; Oberkofler, H.; Lechenauer, E.; Simon, B.; Unger, A.; Kandler, D.; Ebner, C.; Prillinger, H.; Kraft, D.; Breitenbach, M. Molecular cloning of major and minor allergens of Alternaria alternata and Cladosporium herbarum. Mol. Immunol. 1995, 32, 213-227. [CrossRef]

96. Kurup, V.P.; Shen, H.D.; Vijay, H. Immunobiology of fungal allergens. Int. Arch. Allergy Immunol. 2002, 129, 181-188. [CrossRef] [PubMed]

97. Sevinc, M.S.; Kumar, V.; Abebe, M.; Casley, W.L.; Vijay, H.M. Isolation and characterization of a cDNA clone encoding one IgE-binding fragment of Penicillium brevicompactum. Int. Arch. Allergy Immunol. 2005, 138, 12-20. [CrossRef] [PubMed]

98. Mayer, C.; Appenzeller, U.; Seelbach, H.; Achatz, G.; Oberkofler, H.; Breitenbach, M.; Blaser, K.; Crameri, R. Humoral and cell-mediated autoimmune reactions to human acidic ribosomal P2 protein in individuals sensitized to Aspergillus fumigatus p2 protein. J. Exp. Med. 1999, 189, 1507-1512. [CrossRef] [PubMed]

99. Zhang, L.; Muradia, G.; Curran, I.H.; Rode, H.; Vijay, H.M. A cDNA clone coding for a novel allergen, cla $h$ iii, of Cladosporium herbarum identified as a ribosomal P2 protein. J. Immunol. 1995, 154, 710-717. [PubMed]

100. Hoff, M.; Ballmer-Weber, B.K.; Niggemann, B.; Cistero-Bahima, A.; San Miguel-Moncin, M.; Conti, A.; Haustein, D.; Vieths, S. Molecular cloning and immunological characterisation of potential allergens from the mould Fusarium culmorum. Mol. Immunol. 2003, 39, 965-975. [CrossRef]

101. Saxena, S.; Madan, T.; Muralidhar, K.; Sarma, P.U. cDNA cloning, expression and characterization of an allergenic 13 ribosomal protein of Aspergillus fumigatus. Clin. Exp. Immunol. 2003, 134, 86-91. [CrossRef] [PubMed]

102. Tang, R.B.; Chen, Y.S.; Chou, H.; Lee, S.S.; Tai, H.Y.; Shen, H.D. cDNA cloning and immunologic characterization of a novel EF-1beta allergen from Penicillium citrinum. Allergy 2005, 60, 366-371. [CrossRef] [PubMed] 
103. Shen, H.D.; Choo, K.B.; Lee, H.H.; Hsieh, J.C.; Lin, W.L.; Lee, W.R.; Han, S.H. The 40-kilodalton allergen of Candida albicans is an alcohol dehydrogenase: Molecular cloning and immunological analysis using monoclonal antibodies. Clin. Exp. Allergy 1991, 21, 675-681. [CrossRef] [PubMed]

104. Westwood, G.S.; Huang, S.W.; Keyhani, N.O. Molecular and immunological characterization of allergens from the entomopathogenic fungus Beauveria bassiana. Clin. Mol. Allergy 2006, 4, 12. [CrossRef] [PubMed]

105. Simon-Nobbe, B.; Probst, G.; Kajava, A.V.; Oberkofler, H.; Susani, M.; Crameri, R.; Ferreira, F.; Ebner, C.; Breitenbach, M. IgE-binding epitopes of enolases, a class of highly conserved fungal allergens. J. Allergy Clin. Immunol. 2000, 106, 887-895. [CrossRef] [PubMed]

106. Lai, H.Y.; Tam, M.F.; Tang, R.B.; Chou, H.; Chang, C.Y.; Tsai, J.J.; Shen, H.D. cDNA cloning and immunological characterization of a newly identified enolase allergen from Penicillium citrinum and Aspergillus fumigatus. Int. Arch. Allergy Immunol. 2002, 127, 181-190. [CrossRef] [PubMed]

107. Ishiguro, A.; Homma, M.; Torii, S.; Tanaka, K. Identification of Candida albicans antigens reactive with immunoglobulin e antibody of human sera. Infect Immun. 1992, 60, 1550-1557. [PubMed]

108. Mason, A.B.; Buckley, H.R.; Gorman, J.A. Molecular cloning and characterization of the Candida albicans enolase gene. J. Bacteriol. 1993, 175, 2632-2639. [CrossRef] [PubMed]

109. Sharma, V.; Gupta, R.; Jhingran, A.; Singh, B.P.; Sridhara, S.; Gaur, S.N.; Arora, N. Cloning, recombinant expression and activity studies of a major allergen "enolase" from the fungus Curvularia lunata. J. Clin. Immunol. 2006, 26, 360-369. [CrossRef] [PubMed]

110. Holland, M.J.; Holland, J.P.; Thill, G.P.; Jackson, K.A. The primary structures of two yeast enolase genes. Homology between the $5^{\prime}$ noncoding flanking regions of yeast enolase and glyceraldehyde-3-phosphate dehydrogenase genes. J. Biol. Chem. 1981, 256, 1385-1395. [PubMed]

111. Chou, H.; Tam, M.F.; Lee, S.S.; Tai, H.Y.; Chang, C.Y.; Chou, C.T.; Shen, H.D. A vacuolar serine protease (rho $\mathrm{m} 2$ ) is a major allergen of Rhodotorula mucilaginosa and belongs to a class of highly conserved pan-fungal allergens. Int. Arch. Allergy Immunol. 2005, 138, 134-141. [CrossRef] [PubMed]

112. Muthuvel, A.; Rajamani, R.; Senthilvelan, M.; Manikandan, S.; Sheeladevi, R. Modification of allergenicity and immunogenicity of formate dehydrogenase by conjugation with linear mono methoxy polyethylene glycol: Improvement in detoxification of formate in methanol poisoning. Clin. Chim. Acta 2006, 374, 122-128. [CrossRef] [PubMed]

113. Schneider, P.B.; Denk, U.; Breitenbach, M.; Richter, K.; Schmid-Grendelmeier, P.; Nobbe, S.; Himly, M.; Mari, A.; Ebner, C.; Simon-Nobbe, B. Alternaria alternata NADP-dependent mannitol dehydrogenase is an important fungal allergen. Clin. Exp. Allergy 2006, 36, 1513-1524. [CrossRef] [PubMed]

114. Simon-Nobbe, B.; Denk, U.; Schneider, P.B.; Radauer, C.; Teige, M.; Crameri, R.; Hawranek, T.; Lang, R.; Richter, K.; Schmid-Grendelmeier, P.; et al. NADP-dependent mannitol dehydrogenase, a major allergen of Cladosporium herbarum. J. Biol. Chem. 2006, 281, 16354-16360. [CrossRef] [PubMed]

115. Onishi, Y.; Kuroda, M.; Yasueda, H.; Saito, A.; Sono-Koyama, E.; Tunasawa, S.; Hashida-Okado, T.; Yagihara, T.; Uchida, K.; Yamaguchi, H.; et al. Two-dimensional electrophoresis of Malassezia allergens for atopic dermatitis and isolation of Mal $\mathrm{f} 4$ homologs with mitochondrial malate dehydrogenase. Eur. J. Biochem. 1999, 261, 148-154. [CrossRef] [PubMed]

116. De Vouge, M.W.; Thaker, A.J.; Zhang, L.; Muradia, G.; Rode, H.; Vijay, H.M. Molecular cloning of IgE-binding fragments of Alternaria alternata allergens. Int. Arch. Allergy Immunol. 1998, 116, 261-268. [CrossRef] [PubMed]

117. Zhang, L.; Muradia, G.; De Vouge, M.W.; Rode, H.; Vijay, H.M. An allergenic polypeptide representing a variable region of HSP 70 cloned from a cDNA library of Cladosporium herbarum. Clin. Exp. Allergy 1996, 26, 88-95. [CrossRef] [PubMed]

118. Shen, H.D.; Au, L.C.; Lin, W.L.; Liaw, S.F.; Tsai, J.J.; Han, S.H. Molecular cloning and expression of a Penicillium citrinum allergen with sequence homology and antigenic crossreactivity to a HSP 70 human heat shock protein. Clin. Exp. Allergy 1997, 27, 682-690. [CrossRef] [PubMed]

119. Bowyer, P.; Denning, D.W. Genomic analysis of allergen genes in Aspergillus spp: The relevance of genomics to everyday research. Med. Mycol. 2007, 45, 17-26. [CrossRef] [PubMed]

120. Andersson, A.; Rasool, O.; Schmidt, M.; Kodzius, R.; Fluckiger, S.; Zargari, A.; Crameri, R.; Scheynius, A. Cloning, expression and characterization of two new IgE-binding proteins from the yeast Malassezia sympodialis with sequence similarities to heat shock proteins and manganese superoxide dismutase. Eur. J. Biochem. 2004, 271, 1885-1894. [CrossRef] [PubMed] 
121. Kumar, A.; Reddy, L.V.; Sochanik, A.; Kurup, V.P. Isolation and characterization of a recombinant heat shock protein of Aspergillus fumigatus. J. Allergy Clin. Immunol. 1993, 91, 1024-1030. [CrossRef]

122. Crameri, R.; Faith, A.; Hemmann, S.; Jaussi, R.; Ismail, C.; Menz, G.; Blaser, K. Humoral and cell-mediated autoimmunity in allergy to Aspergillus fumigatus. J. Exp. Med. 1996, 184, 265-270. [CrossRef] [PubMed]

123. Fluckiger, S.; Scapozza, L.; Mayer, C.; Blaser, K.; Folkers, G.; Crameri, R. Immunological and structural analysis of IgE-mediated cross-reactivity between manganese superoxide dismutases. Int. Arch. Allergy Immunol. 2002, 128, 292-303. [CrossRef] [PubMed]

124. Limacher, A.; Kloer, D.P.; Fluckiger, S.; Folkers, G.; Crameri, R.; Scapozza, L. The crystal structure of Aspergillus fumigatus cyclophilin reveals 3D domain swapping of a central element. Structure 2006, 14, 185-195. [CrossRef] [PubMed]

125. Shankar, J.; Singh, B.P.; Gaur, S.N.; Arora, N. Recombinant glutathione-s-transferase a major allergen from Alternaria alternata for clinical use in allergy patients. Mol. Immunol. 2006, 43, 1927-1932. [CrossRef] [PubMed]

126. Fukutomi, Y.; Taniguchi, M. Sensitization to fungal allergens: Resolved and unresolved issues. Allergol. Int. 2015, 64, 321-331. [CrossRef] [PubMed]

127. Petersen, T.N.; Brunak, S.; von Heijne, G.; Nielsen, H. Signalp 4.0: Discriminating signal peptides from transmembrane regions. Nat. Methods 2011, 8, 785-786. [CrossRef] [PubMed]

128. McCotter, S.W.; Horianopoulos, L.C.; Kronstad, J.W. Regulation of the fungal secretome. Curr. Genet. 2016, 62, 533-545. [CrossRef] [PubMed]

129. Kaiser, C.A.; Schekman, R. Distinct sets of sec genes govern transport vesicle formation and fusion early in the secretory pathway. Cell 1990, 61, 723-733. [CrossRef]

130. Baker, D.; Hicke, L.; Rexach, M.; Schleyer, M.; Schekman, R. Reconstitution of sec gene product-dependent intercompartmental protein transport. Cell 1988, 54, 335-344. [CrossRef]

131. Deshaies, R.J.; Schekman, R. A yeast mutant defective at an early stage in import of secretory protein precursors into the endoplasmic reticulum. J. Cell Biol. 1987, 105, 633-645. [CrossRef] [PubMed]

132. Novick, P.; Schekman, R. Secretion and cell-surface growth are blocked in a temperature-sensitive mutant of Saccharomyces cerevisiae. Proc. Natl. Acad. Sci. USA 1979, 76, 1858-1862. [CrossRef] [PubMed]

133. Bao, Z.; Xiao, H.; Liang, J.; Zhang, L.; Xiong, X.; Sun, N.; Si, T.; Zhao, H. Homology-integrated CRISPR-Cas (Hi-CRISPR) system for one-step multigene disruption in Saccharomyces cerevisiae. ACS Synth Biol. 2015, 4, 585-594. [CrossRef] [PubMed]

134. Giaever, G.; Nislow, C. The yeast deletion collection: A decade of functional genomics. Genetics 2014, 197, 451-465. [CrossRef] [PubMed]

135. Si, T.; Chao, R.; Min, Y.; Wu, Y.; Ren, W.; Zhao, H. Automated multiplex genome-scale engineering in yeast. Nat. Commun. 2017, 8, 15187. [CrossRef] [PubMed]

136. Hughes, T.R.; Marton, M.J.; Jones, A.R.; Roberts, C.J.; Stoughton, R.; Armour, C.D.; Bennett, H.A.; Coffey, E.; Dai, H.; He, Y.D.; et al. Functional discovery via a compendium of expression profiles. Cell 2000, 102, 109-126. [CrossRef]

137. Miura, N.; Shinohara, M.; Tatsukami, Y.; Sato, Y.; Morisaka, H.; Kuroda, K.; Ueda, M. Spatial reorganization of Sacharomyces cerevisiae enolase to alter carbon metabolism under hypoxia. Eukaryot Cell 2013, 12, 1106-1119. [CrossRef] [PubMed]

138. Gyorgy, B.; Hung, M.E.; Breakefield, X.O.; Leonard, J.N. Therapeutic applications of extracellular vesicles: Clinical promise and open questions. Annu. Rev. Pharmacol. Toxicol. 2015, 55, 439-464. [CrossRef] [PubMed]

(C) 2018 by the authors. Licensee MDPI, Basel, Switzerland. This article is an open access article distributed under the terms and conditions of the Creative Commons Attribution (CC BY) license (http://creativecommons.org/licenses/by/4.0/). 